\title{
Rede de apoio e suporte emocional no enfrentamento da Diabetes Mellitus por gestantes
}

\author{
Ana Cristina Barros da Cunba* \\ Camila Dias Marques** \\ Carolina Peres de Lima***
}

\begin{abstract}
Resumo
O objetivo deste estudo foi investigar o enfrentamento de gestantes com Diabetes Mellitus (DM) que contavam ou não com suporte emocional da rede de apoio (companheiro, mãe, amiga) durante seu pré-natal. Uma amostra de 21 gestantes atendidas na Maternidade escola da UFRJ respondeu um Protocolo de dados gerais e a Escala Modos de Enfrentamento de Problemas. Metade delas era regularmente acompanhada nas consultas pré-natais por alguém da rede de apoio. Independente delas terem tido suporte emocional, a maioria das gestantes utilizava estratégias de enfrentamento "focadas no problema", caracterizada pela aproximação do estressor (DM) para reavaliar o problema e vivenciar mais positivamente a gravidez de risco. Embora a rede de apoio não ofereça suporte emocional durante o pré-natal, mulheres com risco gestacionais, como a DM, podem usar outros apoios, como o instrumental ou financeiro, para auxiliá-las no enfrentamento da sua condição de vulnerabilidade durante a gravidez.

Palavras-chave: coping; suporte social emocional; diabetes mellitus; gravidez.
\end{abstract}

\section{Support network and emotional support in the coping with diabetes mellitus by pregnant women}

\begin{abstract}
The objective of this study was to investigate the coping strategies of pregnant women with Diabetes Mellitus (DM) who had or not emotional support from her support network (partner, mother, friend) during her prenatal care. A sample of 21 pregnant women attended at the Maternidade escola of UFRJ answered a General data protocol and the Ways of Coping with Problems Scale. Half of them were regularly followed by a member of their support network during her prenatal appointments. Regardless those women have had an emotional support, the majority used "problem-focused" coping strategies, characterized by the approaching with the stressor (DM) to re-evaluated their problem, and to experience their at-risk pregnancy more positively. Even though the support network does not offer an emotional support during the prenatal care, the women with pregnancy risks, like DM, can use others supports (instrumental or financial support) to cope with their vulnerable condition during the pregnancy.

Key-words: coping; social-emotional support; diabetes mellitus; pregnancy.
\end{abstract}

* Instituto de Psicologia; Maternidade escola, Universidade Federal do Rio de Janeiro; Programa de pós-graduação em Psicologia, Universidade Federal do Espirito Santo.

** Universidade Federal do Rio de Janeiro

***Graduação em Psicologia pela UFRJ. Especialista em Residência Multiprofissional, INCA. Mestrado em Saúde Coletiva UFRJ. 


\section{CAROLINA PERES DE LIMA}

\section{Introdução}

Dentre as diferentes situações de vulnerabilidade decorrentes de fatores de risco gestacional, destaca-se a gravidez com Diabetes Mellitus (DM) e $\backslash$ ou Diabetes Mellitus Gestacional (DMG). Particularmente, a DMG representa uma intolerância à glicose com aumento das taxas glicêmicas da gestante, diagnosticada pela primeira vez durante a gravidez a partir da $25^{a}$ semana de gestação (Sociedade Brasileira de Diabetes - SBD, 2014). Associada a altos índices de morbidade perinatal, principalmente macrossomia e malformações fetais (Almirón, Gamarra, \& González, 2005; Chaves Netto \& Sá, 2015), tem prevalência de $3 \%$ a $13 \%$ dentre mulheres grávidas e vem crescendo em proporções semelhantes à do Diabetes Mellitus tipo 2 (DM2) (SBD, 2014). No Brasil, estima-se uma prevalência de 2,4\% a 7,2\% de DMG (SBD, 2014; Sociedade Brasileira de Endocrinologia e Metabologia - SBEM, 2008), com complicações e efeitos adversos no período pré-natal e neonatal devido ao controle inadequado das taxas glicêmicas. Além disso, existe o risco da mulher que tem diabetes na gestação desenvolver DM2 após o parto e seu bebê, também, ter a doença (SBD, 2014).

Sabe-se que a gravidez, em geral, é um momento de grandes alegrias e expectativas, mas que pode também gerar na mulher um estado emocional de insegurança, medo e angústias em relação à sua saúde e a do seu bebê. Quando a gravidez é de risco, como a gestação com diabetes, esse estado emocional pode se agravar e causar grande instabilidade emocional e estresse na gestante e seus familiares (Araújo, Pessoa, Damasceno, \& Zanett, 2013). Somado a isso, estudos mostram que a DMG é fator de impacto na saúde mental da gestante com repercussões para sua família. Angústia e temores baseados na crença e medo de permanecerem diabéticas, por exemplo, influenciam negativamente no controle da diabetes e da própria gestação (Castillo \& Vasquez, 2006). Além disso, a DM é fator gerador de estresse e ansiedade em gestantes, sobretudo devido ao rigoroso tratamento que pode incluir procedimentos dolorosos, como a aplicação de insulina injetável diariamente (Marquesim, 2013; Morrison, Lowe, \& Collins, 2014). Tudo isso associado às severas restrições alimentares imposta a gestante resultam em riscos psíquicos para ela e desorganização da sua dinâmica familiar (Almirón, Gamarra \& Gonzalez, 2005).

Nessa perspectiva, é relevante o estudo do estado emocional decorrente desse tipo de vulnerabilidade biológica e psicológica a fim de investigar como a gestante lida com a diabetes e quais estratégias são utilizadas por ela para enfrentar sua condição. Para discutir tais ques- tões, propomos o conceito de coping $^{1}$, levando em consideração que todo indivíduo apresenta formas variadas de enfrentamento (coping) frente a diferentes situações de estresse. O conceito de coping é definido por Lazarus e Folkman (1984) como um processo de "constantes mudanças cognitivas e comportamentais na tentativa de administrar demandas especificas, internas e/ ou externas, que são avaliadas pelo sujeito como excedendo ou sobrecarregando os recursos pessoais" (p. 141). Ainda que existam diferentes modelos de estudo do coping, o presente trabalho adota o modelo de Folkman e Lazarus (1980) que envolve quatro premissas principais: (a) o coping é um processo ou uma interação que ocorre entre o indivíduo e o ambiente; (b) a sua função diz respeito ao manejo de uma situação estressora, ao invés do controle ou domínio da mesma; (c) os processos de coping pressupõem a noção de avaliação, ou seja, como o fenômeno é percebido, interpretado e cognitivamente representado mentalmente pelo indivíduo; (d) o processo de coping constitui-se como uma mobilização de esforços, por meio dos quais os indivíduos empreendem esforços cognitivos e comportamentais para administrar (reduzir, minimizar ou tolerar) as demandas internas ou externas que surgem da sua interação com o ambiente e seus estressores.

Dessa forma, o foco do coping se centra no que a pessoa está fazendo ou pensando no momento presente, em contraste com o que ela faz ou deveria fazer. Logo, pensamentos e ações de coping são sempre dirigidos para condições presentes e particulares e as estratégias podem mudar de um momento para outro, durante os diferentes momentos da situação estressora (Lazarus \& Folkman, 1984). Recursos pessoais tanto quanto sócio ecológicos são considerados no processo de coping que inclui estilos de enfrentamento individuais. Tanto aspectos individuais (características pessoais, saúde física, crenças ideológicas etc.), quanto aspectos do contexto ecológico do indivíduo (características familiares, redes de suporte social e recursos econômicos, por exemplo) são fundamentais para a compreensão sobre como o estresse afeta a vida das pessoas, tanto positiva quanto negativamente (Aldwin, 2010). A disponibilidade destes recursos influencia a avaliação do estressor e determina quais estratégias de coping serão utilizadas (Antoniazzi, Dell'Aglio, \& Bandeira, 1998).

$\mathrm{Na}$ discussão sobre coping inclui-se ainda a ideia de equilíbrio entre risco e proteção. No processo de coping o binômio "fatores de risco X fatores de proteção" deve

1 Considera-se que o termo coping é o correlato em inglês do termo enfrentamento, escolheu-se usar ambos os termos de forma equivalente. 
ser analisado em cada situação de forma singular, já que não é possível determinar uma relação direta de causa e efeito entre eles. Ainda que de um lado eventos estressantes (ameaças, perigos etc.) e condições adversas levem o indivíduo à vulnerabilidade física e psicológica, por outro os recursos pessoais e sócio ecológicos podem representar forças, competências e capacidades individuais de reação e de enfrentamento frente aos estressores (Aldwin, 2010).

Algumas variáveis são consideradas fatores de proteção indiscutíveis, como por exemplo, o suporte social. O suporte social é um construto multidimensional e complexo, mensurável através de conceitualizações objetivas e subjetivas, que variam em termos das diferentes definições adotadas para sua construção, bem como da nomeação de suas dimensões (Baptista, Baptista \& Torres, 2006). O suporte social se refere aos recursos materiais e psicológicos que as pessoas têm acesso por meio de suas redes sociais ${ }^{2}$ (Siqueira, 2008). O suporte social pode ser avaliado pela observação dos recursos que o indivíduo dispõe em seu meio, como, as redes de apoio (serviços e pessoas). Além disso, o suporte social também tem sido associado às diversas outras medidas, tais como competência pessoal, comportamentos de adesão a tratamentos de saúde, estratégias de enfrentamento, percepção de controle, senso de estabilidade, percepção de suporte emocional e bem-estar psicológico (Siqueira, 2008).

De forma geral, o suporte social é um processo interativo que inclui diferentes tipos de auxílio obtidos das redes de apoio, que variam desde o emocional até o instrumental (ajuda em tarefas domésticas, por ex.) ou financeiro (Siqueira, 2008). De acordo com Siqueira (2008), O suporte social emocional diz respeito à relação que as pessoas estabelecem umas com as outras e que servem de ajuda ao outro, incluindo desde dar conselhos e ouvir problemas até mostrar-se empático e confiável, o que pode ser percebido como expressão de carinho, cuidado e preocupação pelo outro. O suporte social emocional é um tipo de recurso oferecido pela rede de apoio. Por sua vez, define-se a rede de apoio como a disponibilidade de apoio e reforço por pessoas significativas ou sistemas diante de situações críticas, oferecidos através de estratégias como carinho, encorajamento ou assistência (Brito \& Koller, 1999).

Em períodos especialmente difíceis da vida, suportes social e emocional adequados podem proporcionar apoio e ajuda no enfrentamento de condições de vulnerabilida-

2 O conceito de "redes sociais" pode ser compreendido como um uma configuração de vínculos interpessoais entrecruzados conectados as ações das pessoas e às instituições sociais (Acioli, 2007). de. Na gravidez, período de particular instabilidade emocional, condições de vulnerabilidade física e psicológica podem ser minimizadas quando a mulher conta com uma rede de apoio e de proteção que a ajude na prevenção aos riscos à sua saúde mental e no enfrentamento de situações de estresse. Nesse sentido, o suporte social, principalmente de ordem emocional, tem sido considerado importante fator de proteção às adversidades decorrentes de uma gestação de risco, como a gravidez com diabetes. Estudo sobre suporte social referido, ansiedade, depressão e estresse revelou que gestantes com diabetes apresentam maiores níveis de ansiedade e depressão e de sintomas de estresse quando contam com menor suporte social (Viviani, 2013).

Sentimentos de angústia e medo podem ter peso redobrado para a gestante com diabetes, que convive com o risco de gerar um bebê com algum tipo de malformação ou que pode mesmo vir a óbito (Chaves Netto \& Sá, 2015). É importante conhecer como essas gestantes enfrentam esse risco na gravidez a fim de auxiliar os profissionais de saúde a planejar uma abordagem educativa acolhedora, que facilite o controle glicêmico e garanta o sucesso da gestação (Silva, Santos \& Parada, 2004). No entanto, a literatura sobre enfrentamento da diabetes na gestação é escassa, quando se faz necessário a realização de pesquisas que forneçam evidências cientificas sobre o tema para subsidiar a prática de atenção à saúde perinatal e mental desse público especifico.

Em geral, os estudos sobre coping e gravidez têm discutido sobre o enfrentamento dos medos da gestação de uma forma geral (Carmona-Monge, Marín-Morales, Peñacoba-Puente, Carretero-Abellán \& Moreno-Moure, 2012), especialmente de gestantes de risco (Villarreal, Villarreal, \& Rodriguez, 2013) e com diagnóstico de malformações congênitas (Vasconcelos \& Petean, 2009), ou mais especificamente em casos de cardiopatias fetais (Benute; Nonnenmacher; Evangelista; Lopes; \& Zugaib, 2011). Especificamente sobre a gestação com diabetes os poucos estudos sobre coping investigam a ocorrência de fatores psicológicos associados, como a depressão pós-parto, ou analisam a vivência feminina da gestação com diabetes (Araújo Pessoa, Damasceno, \& Zanett, 2013; Nicklas; Miller; Zera; Davis; Levkoff; \& Seely, 2013; Morrison; Lowe; \& Collins, 2014), sem discutir a influência de recursos do contexto sócio ecológico no enfrentamento da diabetes na gestação, objetivo principal do nosso estudo.

No contexto da gestação com diabetes, a vulnerabilidade física e psicológica que a mulher e sua família estão 


\section{CAROLINA PERES DE LIMA}

expostos resulta incondicionalmente em estresse. Para lidar com esse estresse a mulher grávida poderia contar com uma rede de apoio que oferecesse a ela o suporte emocional necessário. Esse suporte as ajudaria a escolher estratégias de enfrentamento mais resolutivas para responder as demandas da situação, o que, por sua vez, facilitaria uma vivência mais positiva desse processo singular de gerar uma vida quando se está "doente". Diante disso, nossa hipótese de pesquisa foi de que existem diferentes dispositivos do contexto sócio ecológico da gestante que podem favorecer o seu processo de enfrentamento (coping) da diabetes, dentre esses o suporte social emocional oferecido pela sua rede de apoio. No entanto, nos questionamos se existem diferenças nas estratégias de enfrentamento adotadas pelas gestantes para manejar o estresse decorrente da gravidez de risco com diabetes quando elas não contam com esse tipo de suporte durante seu acompanhamento médico pré-natal. Respostas para questões como essa podem auxiliar os profissionais de saúde a compreender melhor o processo de enfrentamento da diabetes na gestação e, por conseguinte, auxiliar no planejamento de uma prática assistencial voltada para ajudar essas gestantes a enfrentar de forma mais positiva sua condição de risco e vivenciar plenamente sua gravidez. Com base nisso e na hipótese inicialmente formulada, o objetivo desse estudo foi investigar estratégias de enfrentamento (coping) de gestantes com diabetes mellitus que contavam ou não com suporte social emocional de sua rede de apoio direta, presença de companheiro, mãe, amiga etc., durante suas consultas de acompanhamento pré-natal realizado em uma Maternidade-Escola, na cidade do Rio de Janeiro.

\section{Método}

Trata-se de um estudo descritivo exploratório realizado durante um período de três meses $^{3}$, quando participaram 21 gestantes diagnosticadas com DMG. As participantes tinham média de idade de 28 anos e eram atendidas na Unidade de Transtornos Endocrino-metabólicos (UTEN) da Maternidade escola da Universidade Federal do Rio de Janeiro. Do total, nove declararam contar com suporte social emocional, 12 não contavam com esse tipo de suporte durante seu atendimento pré-natal. Com base nisso, as participantes foram divididas em dois grupos: 1) com suporte social emocional (GA

O estudo foi realizado por período de três meses que corresponde ao tempo médio de acompanhamento das gestantes com diabetes no ambulatório especializado em DMG, haja visto que a DMG é diagnosticada entre o $2^{\circ}$ e $3^{\circ}$ trimestre gestacional.
- Grupo com Acompanhante): $n=09$; e 2) sem suporte social emocional (GSA - Grupo Sem Acompanhante): $n=$ 12. Para essa pesquisa foi definido como suporte social emocional a presença de um membro da rede de apoio da gestante (companheiro, mãe, uma amiga etc.), que a acompanhava durante as consultas e exames realizados durante seu acompanhamento pré-natal na instituição e a quem a gestante se referia ser uma pessoa que prestava suporte social emocional, em acordo com a definição de Siqueira (2008).

Todas as gestantes estavam na $26^{\mathrm{a}}$ semana ou mais e frequentavam as consultas pré-natais na UTEM uma vez por semana. $\mathrm{O}$ atendimento na UTEM é conduzido por equipe multiprofissional que conta com médicos (obstetra e endocrinologista), nutricionistas, enfermeiros e psicólogos. Todos os atendimentos especializados faziam parte da rotina da UTEM e eram realizados seguido protocolo assistencial da instituição. As participantes eram abordadas para a pesquisa durante a espera para o atendimento pré-natal, quando era identificado se eles estavam acompanhadas ou não por membro de sua rede de apoio que participaria nas consultas. Após serem esclarecidas sobre os objetivos da pesquisa e aceitarem participar, elas assinavam um Termo de Consentimento Livre e Esclarecido, aprovado pelo Comitê de Ética da instituição (CAEE n. 0033.0.361.361-9 de 28 de junho de 2010).

Todas responderam a um Protocolo de dados gerais, criado para a pesquisa para caracterização do perfil psicossocial, e a EMEP, Escala Modos de Enfrentamento de Problemas, para identificação das estratégias de enfrentamento. A EMEP é uma escala tipo Likert de 5 pontos $(1=$ Eu nunca faço isso; 2 = Eu faço isso um pouco; 3 $=$ Eu faço isso às vezes; $4=$ Eu faço isso muito; $5=\mathrm{Eu}$ faço isso sempre), cuja versão de Seidl, Tróccoli e Zannon (2001) usada nesse estudo contém 45 itens. Ela classifica os dados em quatro perfis de coping: 1) Enfrentamento focalizado no problema: estratégias comportamentais e cognitivas de aproximação em relação ao estressor, voltadas para o seu manejo e para a reavaliação e resignificação do problema; 2) Enfrentamento focalizado na emoção: estratégias cognitivas e comportamentais de esquiva ou negação, expressão de emoções negativas, auto-culpa e/ ou culpabilização de outros com função paliativa ou de afastamento do estressor; 3) Enfrentamento baseado na busca de práticas religiosas/pensamento fantasioso: pensamentos e comportamentos religiosos ou fantasiosos como modos de enfrentamento e manejo do estressor; 4) Enfrentamento baseado na busca de suporte social: estratégias de busca de apoio social emocional e/ou 
instrumental para lidar com o estressor (Seidl; Tróccoli; \& Zannon, 2001).

A EMEP vem sendo utilizada com populações em diferentes condições de vulnerabilidade, tais como doenças crônicas, cardiovasculares ou endócrinas (Seidl; Tróccoli; \& Zannon, 2001); gestantes com diagnóstico de malformação fetal (Petean \& Vasconcelos, 2009) e pais com filhos com malformações neurológicas (Silva, Girão, \& Cunha, 2016). Todos os estudos que usaram a EMEP visavam compreender quais tipos de estratégias de enfrentamento são adotadas em condições de vulnerabilidade para lidar com estressores específicos. Estudos envolvendo gestantes diabéticas e a avaliação de suas estratégias de enfrentamento utilizando a EMEP não foram encontrados, o que justifica e reafirma a relevância desse estudo.

Os dados do Protocolo de dados gerais foram analisados por frequência de ocorrência das seguintes categorias: estado civil, paridade, trabalho, suporte social emocional, instrumental e financeiro. Com exceção do suporte social, os resultados das gestantes para as demais categorias desse instrumento serão apresentados separadamente por grupo: a) GSA, sem suporte social e emocional, e b) GA, com suporte social emocional. Os dados da EMEP foram processados e analisados de acordo com as normas da própria escala e foram calculadas as medianas de frequência das estratégias a fim de identificar qual a mais prevalente para cada grupo.

\section{Resultados}

Das 12 gestantes do grupo GSA, duas afirmaram serem casadas, uma viúva, uma divorciada, seis solteiras e duas não informaram seu estado civil. Entretanto, todas as gestantes do grupo GSA afirmaram ter companheiro e estar em uma relação estável de no mínimo oito meses. Dentro desse mesmo grupo, três gestantes eram primíparas (primeiro filho) e nove já tinham filhos. Sobre o trabalho, sete gestantes afirmaram trabalhar fora, enquanto cinco gestantes não trabalhavam fora de casa.

No grupo GA $(\mathrm{N}=9)$, cinco afirmaram serem casadas e quatro se declararam solteiras; destas, três afirmaram ter companheiro e estar em uma relação estável de no mínimo oito meses. Apenas uma gestante solteira afirmou não ter companheiro. Dentro desse mesmo grupo, oito gestantes eram primíparas (primeiro filho) e uma já tinha filhos. Sobre o trabalho, cinco delas afirmaram trabalhar fora e quatro não trabalhavam fora de casa.

Do total das 21 participantes, nove gestantes que não trabalhavam (5 do GSA e 4 do GA) contavam com suporte social financeiro proveniente da renda do companheiro ou ajuda financeira de familiares. Observou-se, ainda, que do total de 21 participantes, 14 gestantes (seis do grupo GSA e oito do grupo GA) contavam com suporte social instrumental em casa ao longo da gravidez, principalmente nos afazeres domésticos e nos cuidados com os outros filhos.

Para os dados de coping pela Escala Modos de Enfrentamento de Problemas (EMEP) verificou-se que das gestantes do grupo GA $(n=09)$ ou seja, as que contavam com suporte social emocional durante as consultas, $55,5 \%$ $(n=5)$ adotava de forma mais prevalente estratégias de enfrentamento com "foco no problema"; enquanto que as demais $(n=4)$ usavam estratégias de enfrentamento focadas na "busca de práticas religiosas". Dentre as 12 gestantes do grupo GSA, ou seja, mulheres que não contavam com suporte social emocional porque iam sozinhas nas consultas pré-natais, 58,3\% $(n=7)$ adotavam com maior prevalência estratégias de enfrentamento com "foco no problema". As demais gestantes do grupo usavam estratégias focadas na "busca de práticas religiosas" $(25 \% ; n=3)$ e apenas duas $(16,6 \%)$ utilizavam estratégias focadas na "busca de suporte social" (Tabela 1).

abela 1. Frequência das estratégias de coping mais prevalentes nas gestantes com diabetes avaliadas pela EMEP $(N=21)$.

\begin{tabular}{lll}
\hline Tipo de estratégias de coping & GA & GSA \\
\hline Focalizada no Problema & 5 & 7 \\
Focalizado na Emoção & 0 & 0 \\
Busca de Práticas Religiosas & 4 & 3 \\
Busca de Suporte Social & 0 & 2 \\
\hline TOTAL & 9 & 12 \\
\hline GA=gestantes com acompanhante; GSA=gestantes sem \\
acompanhante
\end{tabular}

Os resultados demonstraram que nenhuma participante de nenhum dos dois grupos (GA ou GSA) utilizava estratégias de enfrentamento "focadas na emoção". Igualmente, as participantes do grupo GA não adotavam estratégias "focadas na busca de suporte social", possivelmente porque já contavam com esse suporte representado pelo acompanhamento de um membro de sua rede de apoio nas consultas do atendimento pré-natal. 


\section{CAROLINA PERES DE LIMA}

\section{Discussão}

Em síntese, as estratégias de enfrentamento "focalizadas no problema" foram as mais prevalentes em ambos os grupos de gestantes (com e sem acompanhante durante o pré-natal). Isto significa que, independente de contarem com o suporte emocional de um membro da sua rede de apoio as acompanhando no atendimento pré-natal, aquelas gestantes estavam engajadas no manejo da sua condição de vulnerabilidade e do estresse decorrente da diabetes. Assim, elas adotavam estratégias que possibilitavam enfrentar essa condição lidando diretamente com o problema, de forma a controlar a situação de ameaça, dano ou desafio representada pelo risco gestacional. Segundo Seidl; Tróccoli e Zannon (2001), essa estratégia é estabelecida através de uma aproximação ativa da pessoa com o estressor por meio de recursos de resolução de problemas e de planejamento do enfrentamento da situação estressante.

Nessa direção, podemos supor que o DMG constitui um fator de estresse na gestação que, agregado aos demais estressores já próprios de toda gravidez, imprime na mulher uma urgência em enfrentar diretamente esse problema com o manejo imediato das demandas da situação a fim de garantir a sua saúde física e prevenir desfechos negativos para sua gravidez. Assim, mesmo que a mulher não possa contar com suporte social, a diabetes exigirá dela focar no problema. Por exemplo, o rígido tratamento que lhe é imposto (Almirón, Gamarra, \& González, 2005; Marquesim, 2013). Questiona-se, entretanto, o custo emocional disso para a gestante e sua família, já que a diabetes gera impacto psicológico importante (Castillo \& Vasquez, 2006; Morrison; Lowe; \& Collins, 2014; Nolan, Mccrone, \& Chertok, 2011), especialmente para aquelas que não contavam com suporte emocional e uma rede de apoio(Viviani, 2013).

Com efeito, as gestantes de ambos os grupos apresentaram um estilo de coping semelhante ao longo do pré-natal, representado pela sua aproximação do estressor para uma resignificação do seu problema, que é a sua condição de risco gestacional decorrente do DMG. Considerando que o processo de coping no modelo proposto por Lazarus e Folkman (1984) pressupõe a noção de interpretação cognitiva e representação mental do fenômeno pela pessoa. Pode-se supor que aquelas gestantes atribuíam um significado à diabetes que não se sobrepunha ao significado da experiência de gestar, o que fez com que elas se focassem na resolução do problema adotando esforços cognitivos e comportamentais que manejassem as demandas internas ou externas próprias da gestação de risco.
Assim sendo, independente do suporte emocional oferecido pelo acompanhante nas consultas, elas buscavam outros recursos para lidar diretamente com o problema da diabetes. Isso pode ser explicado, pelo fato de que as gestantes sem acompanhamento nas consultas do pré-natal (GSA) declararam contar com suporte social instrumental (apoio de algum familiar ou amigos para os afazeres diários) que as ajudava a lidar com as demandas da gestação. Isso era especialmente importante para aquelas que já tinham filhos, que era a grande maioria das gestantes do grupo sem apoio (GSA). Esse dado sugere ainda que esse tipo de suporte, o instrumental, representa uma contribuição prática, importante e suficiente para que elas, mesmo sem acompanhamento no atendimento pré-natal, se sentissem amparadas durante a gestação.

Assim, podemos supor que em alguns casos o suporte social instrumental facilita para que a gestante possa lidar com as questões relacionadas à diabetes e as demandas próprias da gravidez. Este suporte facilitaria o acompanhamento da gestação e a adesão ao tratamento da diabetes, porque permitiria à mulher se dedicar mais integralmente aos cuidados com sua saúde e à prevenção dos desfechos negativos para o desenvolvimento do seu bebê. Este tipo de apoio facilitou, inclusive, a frequência delas às consultas pré-natais e mesmo quando desacompanhadas não se sentiram desamparadas, porque sabiam que poderiam contar com o suporte de sua rede em outras situações.

Importante discutir que, apesar de ter sido observado que nem todas as mulheres eram casadas oficialmente $(\mathrm{n}=06), 16$ delas podiam também contar com a ajuda de seus companheiros, outros familiares ou amigos, quer seja financeiramente (suporte financeiro) ou nos afazeres domésticos (suporte instrumental). Isto pode ter proporcionado a elas uma percepção de suporte, conforto e segurança oferecido pela sua estrutura familiar, o que foi de suma importância nos casos de gestação com diabetes (Castillo \& Vasquez, 2006). Para esses autores, a participação do marido ou familiares nos cuidados da mulher por meio de um apoio explícito e de um suporte adequado às necessidades da gestante é fundamental para o bem-estar não só dela como do casal. Sobre esse aspecto, foi observado que, mesmo não tendo um laço formal de relacionamento porque poucas gestantes eram casadas $(n=6)$, a maioria delas $(n=16)$ possuíam companheiro e podiam contar com a presença e a ajuda, tanto afetiva quanto financeira, durante a gestação. Gestantes de ambos os grupos que não estavam trabalhando de forma remunerada contavam, inclusive, com suporte 
financeiro dos seus parceiros. Tal dado é corroborado por estudo de Moretto \& Lautert (2004), no qual também foi observado que gestantes com diabetes, em geral, contam com o comprometimento de seus companheiros oferecendo-lhes apoio financeiro e também emocional, por meio de uma atitude preocupada com a saúde de suas mulheres e seus bebês.

O contrário também ocorreu, ainda que para apenas duas gestantes do grupo GSA. Para essas mulheres, o apoio do companheiro não existiu, nem em termos de suporte emocional e nem instrumental porque elas se declararam sozinhas no manejo econômico, afazeres da casa e cuidado com os outros filhos. Para estas a estratégia de enfrentamento mais prevalente foi a focado na "busca de suporte social". Possivelmente esse suporte social que buscavam se relacionava ao desamparo experimentado por elas durante a gravidez. Tal resultado da EMEP é confirmado por Seidl; Tróccoli; E Zannon (2001) que a estratégia "busca de suporte social" representa a procura por apoio instrumental, emocional ou de informação para o enfrentamento da situação causadora do estresse. Isto era, exatamente, o que aquelas mulheres declararam necessitar. $\mathrm{O}$ apoio não encontrado por elas no contexto familiar fez com que buscassem esse suporte em outros ambientes, até mesmo no contexto do hospital. A esse respeito cabe destacar que durante a coleta da pesquisa observou-se que as participantes desse ambulatório criavam vínculos de amizade e de confiança entre elas mesmas durante a espera do atendimento. A procura por esse suporte social e emocional entre as próprias gestantes pode também ser indicado de um aspecto do enfrentamento da diabetes e suas questões especificas, que são os sentimentos de angustia e medos próprios desse tipo de gravidez (Araújo et al, 2013; Castillo \& Vasquez, 2006; Morrison; Lowe, \& Collins, 2014; Silva et al. 2004).

A nova rede de apoio criada pela convivência entre as gestantes que compartilhavam a mesma condição de vulnerabilidade e o amparo mútuo construído entre elas podem ser indicados como um mecanismo de proteção à saúde mental da mulher. Estudos comprovam que $\mathrm{o}$ enfrentamento focado na busca de suporte social, tanto quanto o enfrentamento focado no problema, tem sido associado a menores níveis de depressão em mulheres com risco gestacional (Vilarreal, Vilarreal, \& Rodriguez, 2013). Especialmente em situações cujo estressor é um problema de saúde, estratégias de coping "focadas no problema", seguidas de "busca de suporte social" e "práticas religiosas" são as mais identificadas pela EMEP (Seidl; Tróccoli; \& Zannon, 2001), o que também foi observado nesse estudo. Isso respalda o uso dessa escala também para esse tipo de população.

Considerando a diabetes como um fator de risco para estresse e ansiedade na gravidez (Morrison; Lowe; \& Collins, 2014; Viviani, 2013), o suporte social emocional oferecido pelo acompanhante durante as consultas merece atenção. Não se pode duvidar que esse suporte é um importante fator de proteção à saúde mental da mulher grávida com diabetes. Entretanto, no presente estudo os dados apontam que ainda mais importante que o suporte emocional é a mulher poder contar com um suporte instrumental e financeiro que a ampare e ajude a lidar com as demandas ao longo da gravidez e não somente em uma consulta pontual de pré-natal. Isso certamente facilitaria, pois estas gestantes, que vêm sua experiência de gravidez atravessada por uma intercorrência tão grave como a DMG, possam vivenciar de forma mais favorável sua gestação, que é um momento singular na vida da mulher.

Nesse sentido, é importante refletir sobre o que Maldonado (2005) nos convida a pensar, pois o nascimento de um filho é uma experiência familiar. Para que essa experiência seja positiva, é importante que seja oferecida uma assistência pré-natal global que convide a todos os envolvidos na gravidez a participarem direta ou indiretamente da atenção à saúde materno-infantil. Dessa forma, propomos que essa atenção à gestação não se centre apenas na "mulher grávida" ou no binômio mãe-filho, mas que volte o seu olhar para a "família grávida". Dessa forma, envolver a família no processo gravídico-puerperal pode ajudar a minimizar os riscos inerentes à diabetes, fazendo com que a família esteja bem para oferecer o suporte emocional e instrumental necessários para uma boa evolução da gravidez de risco. Esses suportes podem ajudar ainda a prevenir desfechos negativos para a saúde mental da gestante com diabetes, como a ansiedade e o estresse (Morrison; Lowe; \& Collins, 2014; Nolan; Mccrone; \& Chertok, 2011; Viviani, 2013). É sabido que esses riscos psíquicos podem se agravar e dificultar uma vivencia positiva da maternidade, com repercussões inclusive no puerpério. Isso pode ser prevenido quando o suporte da rede de apoio se torna um mecanismo de proteção para vulnerabilidade psicológica que esse risco gestacional confere.

Importante considerar que a estrutura nuclear da família é modificada pela chegada de um bebê. Além da tríade mãe, pai e bebê, nascem também avós, irmãos, tios, e até mesmo os amigos e vizinhos que se veem envolvidos nessa dinâmica. Todos representam a rede de apoio da gestante, a qual pode oferecer para ela o 


\section{CAROLINA PERES DE LIMA}

suporte social necessário à uma boa evolução do ciclo gravídico-puerperal. É fundamental, portanto, valorizar as interações de toda o contexto sócio ecológico da mulher grávida, onde ela reconhece e se apropria dos recursos de coping que lhes serão úteis (Aldwin, 2010). A unidade familiar da gestante tem importante participação do enfrentamento da DMG, na medida em que cada membro desta engrenagem sofre transformações significativas com a presença de um risco gestacional como é a diabetes na gravidez (Moretto \& Lautert, 2004).

O diabetes mellitus como uma doença que tem impacto e afeta de forma multidimensional não só a gestante, como também seus familiares, exige um acolhimento da equipe multiprofissional, que deve fornecer um atendimento especializado e singular de acordo com as peculiaridades de cada paciente. Neste sentido, a Sociedade Brasileira de Diabetes (SBD) menciona, inclusive, ser de extrema importância contar com o acompanhamento de um psicólogo nas situações de estresse, bem como conhecer o desenvolvimento da gravidez em mulheres com diabetes a fim de evitar sobressaltos (SBD, 2014). Se a gravidez já muda toda a dinâmica e configuração familiar, o tratamento da diabetes durante uma gravidez muda completamente a rotina e os hábitos da mulher e sua família. Dessa forma, é extremamente importante a atenção e o cuidado também aos familiares, que passam a representar um importante suporte social de apoio a gestante nesse difícil percurso de uma gravidez de risco.

\section{Considerações Finais}

Nossos dados ainda que preliminares permitem concluir que é importante que a gestante nessa condição possa contar com uma rede de apoio na qual esteja disponível diferentes tipos de recursos, desde o suporte emocional até o instrumental e financeiro. A ajuda de familiares e amigos no cuidado dos outros filhos e da rotina doméstica, assim como a colaboração dos colegas de trabalho para aquelas que têm uma ocupação laboral e que precisam manter medidas de tratamento estressantes, como a insulina injetável durante a jornada de trabalho, são medidas importantes para garantir que a gestante possa se dedicar com mais tranquilidade à vivencia de sua gravidez. Nesse sentido, conhecer sobre o enfretamento (coping) que esse tipo de população utiliza contribui para o planejamento de um acompanhamento e orientação individualizados, com base em suas necessidades e voltados para seus desejos e prioridades. É imprescindível compreender como essas gestantes lidam com uma doença crônica como o diabetes neste período já tão crítico que é a gravidez. Mesmo para aquelas que já eram portadoras de diabetes e aparentemente já conheciam suas limitações, deve ser oferecido um acompanhamento especializado para que a diabetes não resulte em maior estresse e ansiedade que representam riscos psíquicos para a saúde mental da mãe e, consequentemente, repercussões para sua ligação afetiva com seu bebê.

Apontam-se como limitações do estudo a amostra restrita que não permitiu analises estatísticas inferências. Tais analises permitiram investigar, por exemplo, associações entre o suporte emocional e as variáveis sociodemográficas, assim como relações entre as estratégias de coping e os desfechos clinico-obstétricas mais graves. Coloca-se, assim, isso como sugestão para futuros estudos porque entende-se que outras comorbidades associados à diabetes poderiam implicar na adoção de estratégias de enfrentamento diversas para lidar com uma maior gravidade da situação. Também devido a escolha das variáveis de estudo e o delineamento adotado não foi possível verificar a presença de manifestações psíquicas, como a depressão ou a ansiedade na gestação com diabetes, o que permitiria um estudo mais amplo do enfrentamento da diabetes na gestação. Por fim, o perfil da amostra é uma limitação para generalização dos dados, tendo em vista que fizeram parte do estudo somente gestantes que recebiam atendimento especializado em DMG em uma instituição que é um hospital maternidade referência para esse tipo de gestação de risco. Portanto, estes dados não podem ser generalizados para a população de gestantes com DMG.

Por fim, conclui-se que a equipe multiprofissional, incluindo o psicólogo, pode assumir um papel de mediador no processo de enfrentamento do estresse originado pela diabetes quando ela reconhece quais as estratégias de coping são mais frequentemente utilizadas por esse tipo de população. Com isso, os profissionais podem melhor compreender como a gestante lida com a diabetes e, assim, auxiliá-la a enfrentar de forma mais resiliente essa situação de vulnerabilidade. Tal conhecimento certamente sustentaria uma prática humanizada, sensível e preventiva do impacto dos riscos decorrentes das adversidades próprias da gravidez e da diabetes.

\section{Referências:}

Acioli, S. (2007). Redes sociais e teoria social: revendo os fundamentos do conceito. Revista Informação e Informação. Londrina - PR, v. 12, número especial. 2007. Disponível em http://www.uel.br/revistas/uel/index.php/ informacao/article/viewFile/1784/1520

Aldwin, C. (2010). Stress and coping across the lifespan. The Oxford Handbook of Stress, Health, and Coping, 15-34. Doi:10.1093/oxfordhb/9780195375343.013.0002 
Almirón, M. E., Gamarra, S. C., \& González, M. S. (2005). Diabetes Gestacional. Revista de Posgrado de la via Cátedra de Medicina, 152, 23-27.

Antoniazzi, A. S., Dell'Anglio, D. D., \& Bandeira, D. R. (1998). O conceito de coping: uma revisão teórica. Estudos de Psicologia, 3 (2), 273-294.

Araújo, M. F. M.; Pessoa, S. M. F.; Damasceno, M. M. C. \& Zanetti, M. L. (2013). Diabetes gestacional na perspectiva de mulheres grávidas hospitalizadas. Rev Bras Enferm, 66 (2): 222-7.

Baptista, M. N.; Baptista, A. S. D;, \& Torres, E. C. R. (2006). Associação entre suporte social, depressão e ansiedade em gestantes. Psic: revista da Vetor Editora, 7(1), 39-48. Recuperado em 09 de agosto de 2017, de http://pepsic.bvsalud.org/scielo.php?script $=$ sci_arttext\&pid $=$ S1676$-73142006000100006 \& \operatorname{lng}=p t \& t \operatorname{lng}=p t$

Benute, G. R. G.; Nonnenmacher, D.; Evangelista, L. F. M.; Lopes, L. M.; Lucia, M. C. S. \& Zugaib, M. (2011). Cardiopatia fetal e estratégias de enfrentamento. Revista Brasileira de Ginecologia e Obstetrícia, 33 (9), 227-233. Disponível em http://dx.doi.org/10.1590/S0100-72032011000900002

Brito, R. C., \& Koller, S. H. (1999). Desenvolvimento humano e redes de apoio social e afetivo. In: A. M. Carvalho (Org.) O mundo social da criança: natureza e cultura em ação (pp.115-129). São Paulo: Casa do Psicólogo.

Carmona-Monge, F. J.; Marín-Morales, D.; Peñacoba-Puente, C.; Carretero-Abellán, I.; Moreno-Moure, M. A. (2012). Influence of coping strategies in the specific worries of pregnancy. Anales de Psicologia, 338-343.

Castillo, C. A., \& Vásquez, M. L. (2006). El cuidado de si de la embarazada diabética como uma via para asegurar um hijo sano. Texto Contexto Enfermagem. 15 (1), 74-81.

Chaves Netto, H. \& Sá, R. A. M. (2015). Obstetrícia Básica. 2ed. São Paulo: Atheneu.

Folkman, S., \& Lazarus, R.S. (1980). An analysis of coping in a middle-aged community sample. Journal of Health and Social Behavior, 21, 219-239.

Lazarus, R. S., \& Folkman, S. (1984). Stress, appraisal and coping. New York: Springer Publishing Company.

Maldonado, M. T. P. (2005). Aspectos psicológicos da gravidez, do parto e do puerpério. IN: M. T. P. Maldonado. Psicologia da Gravidez: parto e puerpério. (pp. 13-100). Petrópolis, RJ: Vozes.

Marquesim, N.A.Q. (2013). Qualidade de vida, ansiedade e depressão em gestantes diabéticas ou com hiperglicemia. Dissertação de Mestrado. Programa de pós-graduação em tocoginecologia. Faculdade de Medicina de Botucatu, UNESP.

Moretto, V. L.; LautertM L. (2004). Características de gestantes portadoras de diabete melito. Rev Gaúcha Enferm, 25(3):334-45.
Morrison, M. K.; Lowe, J. M. \& Collins, C. E. (2014). Australian women's experiencies of living with gestational diabetes. Women and Birth, 27, 52-57.

Nicklas, J. M.; Miller, L. J.; Zera, C. A.; Davis, R. B.; Levkoff, S. E. \& Seely, E. W. (2013). Factors Associated with Depressive Symptoms in the Early Postpartum Period Among Women with Recent Gestational Diabetes Mellitus. Matern Child Health J, 17, 1665-1672.

Nolan, J. A.; Mccrone, S. \& Chertok, I.R. (2011). The maternal experience of having diabetes in pregnancy. J Am Acad Nurse Pract;23 (11):611-18.

Seidl, E. M. F., Tróccoli, B. T., \& Zannon, C. M. L. C. (2001). Análise Fatorial de Uma Medida de Estratégias de Enfrentamento. Psicologia: Teoria e Pesquisa, 17 (3), 225-234.

Silva, E. H. P.; Girão, E. R. C.; Cunha, A. C. B. (2016). Enfrentamento do pai frente à malformação congênita do filho antes e depois do nascimento. Estudos e Pesquisas em Psicologia, 16 (1), 180-199.

Silva, L., Santos, R. C., \& Parada, C. M. G. L. (2004). Compreendendo o Significado da Gestação para grávidas diabéticas. Revista Latino Americana de Enfermagem, 12 (6), 899-904.

Sociedade Brasileira de Diabetes (SBD). (2014) Diabetes e Gravidez: Disponível em http://www.diabetes.org.br/profissionais/diabetes-gestacional

Sociedade Brasileira de Endocrinologia e Metabologia (SBEM) (2008). Diabetes mellitus gestacional. Rev Assoc Med Bras, 54 (6), 477-80.

Siqueira, M. M. M. (2008). Construção e validação da escala de percepção de suporte social. Psicologia em Estudo, 13 (2), 381-388.

Villarreal, R. V. C.; Villarreal, L. C. P \& Rodriguez, A. P. P. (2013). Relationship between levels of depression and coping strategies in women with gestational risk. (2013). Rev Univ. salud., 15 (2). Disponivel em http://www.scielo. org.co/scielo.php?script $=$ sci_arttext\&pid $=$ S0124-71072013000200006\&ln $\mathrm{g}=$ en\&nrm $=$ iso

Viviani, J. C. (2013). Caracterização psicossocial de gestantes diabéticas em acompanhamento pré-natal em hospital terciário. Dissertação de Mestrado. Ribeirão Preto, SP: Faculdade de Filosofia, Ciências e Letras de Ribeirão Preto.

Vasconcelos, L.; \& Petean, E. B. L. (2009). O impacto da malformação fetal: indicadores afetivos e estratégias de enfrentamento das gestantes. Psicologia, saúde \& doenças, 10 (1), 69-82.

Submetido em: 16-3-2017

Aceito em: $12-9-2017$ 